\title{
Considering cost in primary care
}

I t's a simple question, only five words long, but one asked far too infrequently in primary care: How much does it cost? Dr. Danielle Martin, a family doctor in Toronto, Ontario, was reminded of it recently when she and a resident were deciding which drug to prescribe for a patient. She asked the resident for the names of the cheapest drugs in the class, which were all essentially equivalent. The resident didn't know.

"We looked it up. It took 10 minutes," says Martin, chair of the board of Canadian Doctors for Medicare. "This patient had private drug coverage. It wasn't a question of whether she could afford to pay for the prescription. My point was that this is a consideration we should always take into account. Someone is paying for this drug."

Prescribing expensive drugs when cheaper alternatives exist was but one of many drivers of rising health care costs discussed Nov. 15 at the Family Medicine Forum in Toronto, Ontario, during a session about what family doctors can do to improve the sustainability of Canada's health care system

"Decisions made in primary care affect health system costs by driving up utilization in doctors' visits, diagnostics and drugs - the three D's," says Martin. "What are the factors that play into how we make those decisions?"

Discussing costs can be a touchy matter in primary care as many physicians believe their responsibility is to provide the best care for patients, not to keep Medicare afloat. But "one of the most critical things - maybe the most critical thing when it comes to talking to physicians about sustainability - is not to set those two things up as if they are opposing forces," says Martin. "It's not 'either I do what's good for the system or what's good for my patient.' It's about doing what's best for my patient and then understanding the impact of that on the system."

There are several ways that individual practitioners can contribute to improving Medicare's sustainability, Dr. Monika Dutt, a family physician in Sydney, Nova Scotia, noted during a presentation to the forum, which is co-hosted by the College of Family Physicians of Canada (CFPC),

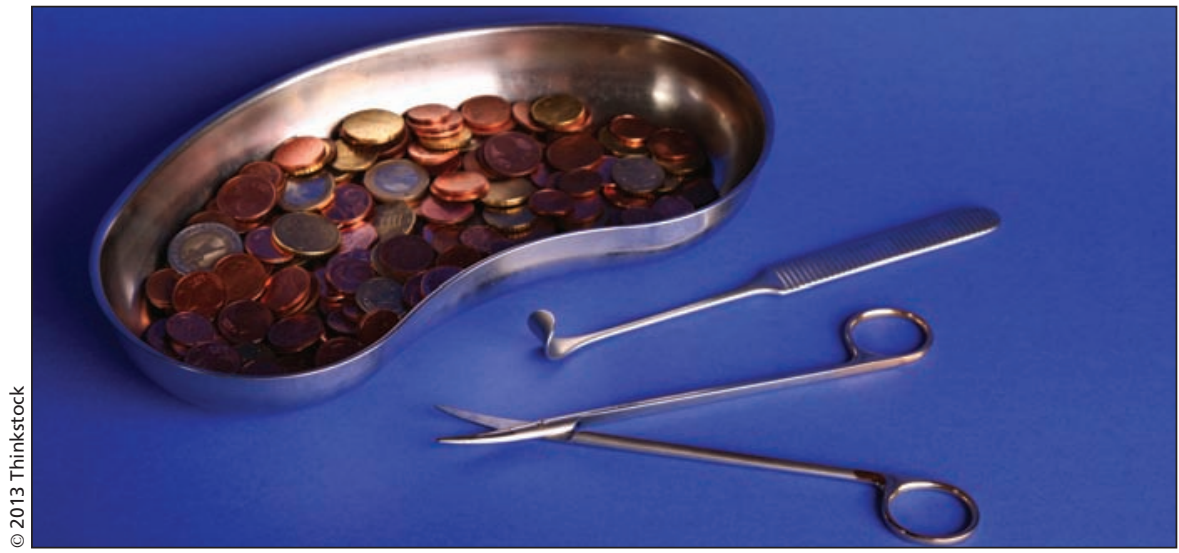

Delisting a medical service often provokes angry reactions.

the Ontario College of Family Physicians and the CFPC's Sections of Teachers and Researchers. They can attempt to reduce the number of unnecessary tests they order. They can include cost-effectiveness in their prescribing decisions. And they can take time to talk with patients about reasonable expectations regarding drugs, tests and other services.

"At the physician level, we need to have a more united voice so that, together, we can improve the system," says Dutt. "It's not about you making less money or me making more money."

A critical step in reducing costs in family medicine is to incorporate costeffectiveness into clinical guidelines that physicians follow in their practices, something few guidelines currently take into consideration, Martin argues. If that were to change, perhaps physicians would prescribe fewer drugs or, in some cases, recommend that patients simply wait (while their conditions are monitored) rather than immediately pursue tests or interventions. "Guidelines are not just about doing stuff. They are also about not doing stuff," she says.

Martin also suggests that there should be "better and more specific guidance on nonpharmacological options in guidelines, not just: Diet and exercise are important and now here are 40 pages on how to prescribe a statin."

Family doctors could also save the health care system a considerable amount of money by resisting the allure of every shiny, new medical device that comes on the market, as difficult as that may be, she adds. "The increasing number of technical options available to us, which is frankly dizzying sometimes, can make it difficult to make rational choices."

But family doctors can't be expected to weigh every financial and medical factor on their own when making decisions, she cautions. It would be overwhelming. That's where things such as checklists, prompts from electronic medical records and automatic substitution of drugs at the pharmacy level come into play. "We need these embedded in the system in which we work, so we are guided down the path that is both clinically effective and cost-effective each time, rather than leaving so much latitude at the clinical level that we are asking the impossible of the clinician - to take into account all these conditions."

Martin also contends that provincial governments can help reduce the burden by taking decisions regarding unnecessary tests or procedures out of the hands of doctors. Though delisting a medical service often provokes angry reactions from patients and physicians alike, it does relieve family doctors from many debates with patients about services with high costs but no clinical benefit, she says. "That simply allows me, as a practitioner, to wash my hands of it and say, sorry, I can't do this test."

"That is a useful thing in my day-today practice," Martin adds. "Instead of me discussing it with each patient, I can say the decision was made at a higher level." - Roger Collier, CMAJ

CMAJ 2013. DOI:10.1503/cmaj.109-4353 